\title{
Similarity solutions for unsteady shear-stress-driven flow of Newtonian and power-law fluids: slender rivulets and dry patches
}

\author{
Y. M. YATIM, ${ }^{1}$ B. R. DUFFY ${ }^{2}$ and S. K. WILSON ${ }^{3}$ \\ Department of Mathematics and Statistics, University of Strathclyde, Livingstone Tower, \\ 26 Richmond Street, Glasgow G1 1XH, U.K.
}

\begin{abstract}
Unsteady flow of a thin film of a Newtonian fluid or a non-Newtonian power-law fluid with power-law index $N$ driven by a constant shear stress applied at the free surface, on a plane inclined at an angle $\alpha$ to the horizontal, is considered. Unsteady similarity solutions representing flow of slender rivulets and flow around slender dry patches are obtained. Specifically, solutions are obtained for converging sessile rivulets $(0<\alpha<\pi / 2)$ and converging dry patches in a pendent film $(\pi / 2<\alpha<\pi)$, as well as for diverging pendent rivulets and diverging dry patches in a sessile film. These solutions predict that at any time $t$ the rivulet and dry patch widen or narrow according to $|x|^{3 / 2}$ and the film thickens or thins according to $|x|$, where $x$ denotes distance down the plane, and that at any station $x$ the rivulet and dry patch widen or narrow like $|t|^{-1}$ and the film thickens or thins like $|t|^{-1}$, independent of $N$.
\end{abstract}

Key words: power-law fluid, thin film, rivulet, dry patch, similarity solution, unsteady flow

\section{Introduction}

Flow problems involving a thin film of fluid subject to pressure and/or shear-stress forces at its free surface due to an external flow occur commonly in practice. A simple example of a flow of this type is the motion of rainwater up the windscreen of a fast moving vehicle; more technologically significant examples include the so-called air-knife coating process, and the icing of aircraft wings when an aircraft passes through a cloud of supercooled water droplets.

There have been many studies of steady flows of such films. For example, King and Tuck [1], using a model proposed by King et al. [2] and based on lubrication and thin-airfoil theories,

\footnotetext{
${ }^{1}$ Current address: School of Mathematical Sciences, Universiti Sains Malaysia, 11800 Penang, Malaysia.

${ }^{2}$ Author to whom correspondence should be addressed. Electronic mail: b.r.duffy@strath.ac.uk

${ }^{3}$ Electronic mail: s.k.wilson@strath.ac.uk
} 
considered a steady two-dimensional ridge of fluid on an inclined plane whose draining due to gravity is resisted by the pressure and shear forces associated with an upward stream of air flowing over its free surface. In a study of rimming flow (that is, flow on the inner surface of a rotating circular cylinder) of a thin film subject to a constant shear stress at its free surface, Villegas-Diaz et al. [3] showed that not only is there a steady solution akin to the well-known solution obtained by Moffatt [4] representing a balance between gravity and shear due to the rotation of the cylinder, but also there can be an additional solution arising because of the surface shear. Steady shear-stress-driven rivulets have been considered by, for example, Wilson and Duffy [5] and Sullivan et al. [6], who studied steady unidirectional flow of a symmetric rivulet of a non-perfectly wetting fluid on a vertical plane and a perfectly wetting fluid on an inclined plane, respectively, subject to a constant longitudinal shear stress at its free surface. They showed that there are five possible types of cross-sectional flow pattern, and investigated the quasi-steady stability of the rivulet; they also determined conditions under which it is energetically favourable for the rivulet to split.

In addition to the above examples of steady flows of thin films subject to external air flow, there have also been studies of unsteady flows of such films. For example, King et al. [2] used their model to investigate surface waves that can occur on an infinite sheet of fluid on an inclined plane subject to pressure and shear forces due to an airflow over it; they deduced that steady waves are possible only for a restricted range of values of mass flux, those of largest (finite) flux being sinusoidal, and those of smallest (non-zero) flux being solitary waves. McKinley et al. [7] studied the spreading of a ridge of fluid and of a droplet on a horizontal plane subject to a non-uniform pressure associated with a jet of air blowing normally onto it, and subsequently McKinley and Wilson [8,9] investigated the linear stability of the ridge and droplet, respectively. Eames et al. [10] obtained similarity solutions describing the effect of a constant surface shear stress due to the flow of an overlying fluid on a spreading viscous gravity current emanating from a point or line source in a horizontal channel; they found that their analytical and numerical solutions are in good agreement with their own experimental results for the cases of a constant volume and a constant flux of fluid released from a point source.

A surface shear stress may be induced not only by an external airflow but also by gradi- 
ents of surface tension induced by, for example, surface-temperature gradients (the well-known thermocapillarity or Marangoni effect). Problems of this type were considered by, for example, Schwartz [11] in his analytical and numerical study of a film drawn up from an infinite bath of fluid onto a vertical plate by thermocapillarity forces induced by a variation in the temperature of the plate, by Holland et al. [12], who obtained similarity solutions describing steady flow of a non-isothermal non-uniform slender rivulet driven by either gravity or a constant shear stress at its free surface subject to thermocapillarity effects, as well as for thermocapillarity-driven rivulets, and more recently by Gatapova and Kabov [13], who considered steady flow in an inclined rectangular microchannel sheared by gas flow with a localised heat source at the bottom of the channel.

There is also a large body of work concerning the effects of surface shear on the behaviour of two-dimensional and three-dimensional drops attached to solid surfaces, including, for example, the work of Dussan V. [14], Li and Pozrikidis [15], Dimitrakopoulos and Higdon [16], Dimitrakopoulos [17], Ding and Spelt [18], and Ding et al. [19].

Pioneering studies on flow around dry patches in a thin film draining down an inclined plane under gravity and surface shear were performed by Hartley and Murgatroyd [20] and Murgatroyd [21], who obtained conditions on the film thickness and flow rate for a dry patch to persist; their theoretical predictions for the shear-driven case were validated numerically and experimentally by Penn et al. [22]. Ponter et al. [23] derived corresponding conditions for a dry patch to persist in a film flowing down a vertical plane in the presence of mass transfer, and showed that their theoretical prediction is in good agreement with their own experiments. Wilson et al. [24] obtained steady similarity solutions for flow around a non-uniform slender dry patch in a thin film draining under gravity on an inclined plane, and subsequently an existence theory for solutions of their mathematical problem was provided by Agarwal and O'Regan [25]. Holland et al. [26] obtained steady similarity solutions for both gravity-driven and constant-shear-stress-driven flow around dry patches in a non-isothermal film subject to thermocapillarity effects, as well as for thermocapillary-driven flow around a dry patch. The shapes and stability of dry patches in a film on an inclined plane were studied experimentally by Podgorski et al. [27, 28] and Rio and Limat [29], who compared their results with a model of 
a dry patch similar to that proposed by Wilson [30] based on a balance between surface tension and gravity. An improved model was studied by Sébilleau et al. [31] which includes the effects of contact-line curvature, hydrostatic pressure and inertia; this is in good agreement with their own experimental results and with those of Podgorski et al. [27].

Most of the work described above concerns flow of a Newtonian fluid; there are somewhat fewer papers on surface-shear-stress-driven flow of a non-Newtonian power-law fluid. For example, Pascal [32] obtained analytical and numerical solutions for the spreading of a fixed volume of a power-law fluid over a slightly denser Newtonian fluid subject to a shear stress at the free surface. Recently, Pascal and D'Alessio [33] investigated the linear stability of the flow of Newtonian and power-law fluids down an inclined plane subject to a prescribed shear stress at the free surface; they found that a shear stress down the plane destabilizes the flow whereas a shear stress up the plane stabilizes it. Wilson et al. [34] obtained similarity solutions describing steady flow of non-uniform slender rivulets of Newtonian and power-law fluids on an inclined plane, driven by either gravity or a constant shear stress at its free surface; their solutions for the two different driving mechanisms are qualitatively similar.

In addition to all the papers described above there is a much bigger literature on purely gravity-driven flows of a thin film, much of which is relevant to the present study. Smith [35] obtained a similarity solution describing steady flow of a sessile rivulet issuing from a point source on an inclined plane, and this was generalised by Wilson and Burgess [36] to the case of a power-law fluid. Perazzo and Gratton [37] obtained a wide class of steady two-dimensional solutions for flow of a power-law fluid on an inclined plane. Smith [38], adapting the analysis of Pattle [39], obtained a similarity solution describing the gravity-driven spreading of a sessile thin film of a constant volume of fluid on a horizontal plane in two or three dimensions; this was generalised by Huppert [40] to the case of a fluid issuing from a time-dependent source, and by Gorodtsov [41] to the case of a power-law fluid. Huppert [42] obtained a similarity solution describing two-dimensional unsteady flow of a thin film down an inclined plane, and Lister [43] obtained similarity solutions describing unsteady flow of a Newtonian fluid down an inclined plane from point and line sources for short times and long times.

Following the approach of our recent work on unsteady gravity-driven flow of a slender 
rivulet of a Newtonian fluid (Yatim et al. [44]), in the present paper we investigate unsteady flow of a slender rivulet of a Newtonian fluid or a non-Newtonian power-law fluid on an inclined plane, and unsteady flow around a slender dry patch in a film of such a fluid, the flow in each case being driven by a constant longitudinal shear stress at its free surface.

\section{Problem formulation}

Consider unsteady three-dimensional flow of a thin film of either a Newtonian fluid with constant density and viscosity or a non-Newtonian power-law fluid with constant density $\rho$ and variable viscosity $\mu=\mu_{0} q^{N-1}$, where $\mu_{0}$ is a constant, $q$ is the local shear rate and $N(>0)$ is the power-law index, on a planar substrate inclined at an angle $\alpha(0 \leq \alpha \leq \pi)$ to the horizontal, driven by a constant shear stress $\tau(>0)$ at its free surface directed down the plane. The power-law fluid is shear thinning when $0<N<1$ and is shear thickening when $N>1$; when $N=1$ the special case of a Newtonian fluid with constant viscosity is recovered. When $0<\alpha<\pi / 2$ the fluid is on the upper side of the inclined plane (the sessile case), and when $\pi / 2<\alpha<\pi$ it is on the underside of the inclined plane (the pendent case). We shall be concerned with both unsteady slender rivulets of a power-law fluid and unsteady slender dry patches in a film of such a fluid.

We adopt Cartesian coordinates $(x, y, z)$ with the $x$ axis down the line of greatest slope and the $z$ axis normal to the substrate, with the substrate at $z=0$, and we denote the free surface profile of the film by $z=h(x, y, t)$, where $t$ denotes time. We restrict attention to flows that are symmetric about $y=0$ (that is, to solutions for which $h$ is even in $y$ ). We denote the (unknown) semi-width of the rivulet or dry patch by $a=a(x, t)$, so that $h=0$ at the contact lines $y= \pm a$; for a rivulet the fluid occupies $|y| \leq a$, whereas for a dry patch the fluid occupies $|y| \geq a$. Figures 1 and 2 show the geometries of the rivulet and dry-patch problems, respectively.

Unsteady flow of an incompressible power-law fluid is governed by the mass and momentum balances

$$
\nabla \cdot \mathbf{u}=0, \quad \rho \frac{D \mathbf{u}}{D t}=-\nabla p+\rho \mathbf{g}+\nabla \cdot(\mu(q) \mathbf{e})
$$


where $\mathbf{u}=(u(x, y, z, t), v(x, y, z, t), w(x, y, z, t))$ and $p=p(x, y, z, t)$ denote velocity and pressure, $\mathbf{g}=g(\sin \alpha, 0,-\cos \alpha)$ denotes gravitational acceleration, e denotes the rate-of-strain tensor, and the local shear rate $q=q(x, y, z, t)$ is given by

$$
q=\left(2 \operatorname{tr}\left(\mathbf{e}^{2}\right)\right)^{\frac{1}{2}}=\left[2 u_{x}^{2}+2 v_{y}^{2}+2 w_{z}^{2}+\left(u_{y}+v_{x}\right)^{2}+\left(u_{z}+w_{x}\right)^{2}+\left(v_{z}+w_{y}\right)^{2}\right]^{\frac{1}{2}} .
$$

These equations are to be solved subject to the boundary conditions of no slip and no penetration at $z=0$ :

$$
u=v=w=0
$$

and balance of stress at $z=h$ :

$$
-p \mathbf{n}+2 \mu \mathbf{e} \cdot \mathbf{n}=-p_{\mathrm{a}} \mathbf{n}+\tau \mathbf{t}-\gamma \kappa \mathbf{n}
$$

where $\mathbf{n}=\left(-h_{x},-h_{y}, 1\right)\left(1+h_{x}^{2}+h_{y}^{2}\right)^{-1 / 2}$ denotes the unit outward normal to the fluid at $z=h$, $\kappa=\nabla \cdot \mathbf{n}$ is the mean curvature of the free surface, $\mathbf{t}=\left(1,0, h_{x}\right)\left(1+h_{x}^{2}\right)^{-1 / 2}$ is the down-plane unit tangent vector to the free surface in the $x, z$ plane, $\gamma$ is the (constant) coefficient of surface tension, and $p_{\mathrm{a}}$ denotes atmospheric pressure. In addition we have the kinematic condition on $z=h:$

$$
h_{t}+\bar{u}_{x}+\bar{v}_{y}=0
$$

and zero-mass-flux conditions at the contact lines $y= \pm a(x, t)$ :

$$
\bar{v}= \pm a_{x} \bar{u}
$$

where the local fluxes $\bar{u}=\bar{u}(x, y, t)$ and $\bar{v}=\bar{v}(x, y, t)$ are defined by

$$
\bar{u}=\int_{0}^{h} u \mathrm{~d} z, \quad \bar{v}=\int_{0}^{h} v \mathrm{~d} z
$$

(see, for example, Young [45]).

For either a thin shear-stress-driven slender rivulet or a slender dry patch in a thin shearstress-driven film we non-dimensionalise and scale according to

$$
\left.\begin{array}{r}
x=\ell x^{*}, \quad y=\epsilon \ell y^{*}, \quad z=\epsilon \delta \ell z^{*}, \quad h=\epsilon \delta \ell h^{*}, \quad a=\epsilon \ell a^{*}, \\
t=\frac{\ell}{U} t^{*}, \quad u=U u^{*}, \quad v=\epsilon U v^{*}, \quad w=\epsilon \delta U w^{*}, \\
p=p_{\mathrm{a}}+\epsilon \delta \rho g \ell|\cos \alpha| p^{*}, \quad q=\left(\frac{\tau}{\mu_{0}}\right)^{\frac{1}{N}} q^{*}, \quad \mu=\mu_{0}\left(\frac{\tau}{\mu_{0}}\right)^{\frac{N-1}{N}} \mu^{*},
\end{array}\right\}
$$


where $\delta \ll 1$ and $\epsilon \ll 1$ are the small aspect ratios in the $y, z$ and $x, y$ planes, respectively, $\ell=\tau / \delta^{2} \rho g|\cos \alpha|$ is the length scale in the $x$ direction, and $U=\left(\tau / \mu_{0}\right)^{1 / N} \epsilon \delta \ell$ is a characteristic velocity down the plane. Dropping the superscript stars for clarity we obtain from (2)

$$
\begin{aligned}
q=\left[u_{z}^{2}+\epsilon^{2} v_{z}^{2}+\delta^{2} u_{y}^{2}\right. & +2 \epsilon^{2} \delta^{2}\left(u_{x}^{2}+v_{y}^{2}+w_{z}^{2}+u_{y} v_{x}+v_{z} w_{y}+u_{z} w_{x}\right) \\
& \left.+\epsilon^{2} \delta^{4} w_{y}^{2}+\epsilon^{4} \delta^{2} v_{x}^{2}+\epsilon^{4} \delta^{4} w_{x}^{2}\right]^{\frac{1}{2}}
\end{aligned}
$$

therefore for the flows with $u_{z} \geq 0$ that will be considered in the present work, the shear rate is given by $q=u_{z}$ at leading order in $\delta$ and $\epsilon$, and hence the viscosity $\mu$ is given by $\mu=u_{z}^{N-1}$ at leading order.

Provided that $\epsilon|\tan \alpha| \ll \delta$ (which requires that $\alpha \neq \pi / 2$ ) and that $R \ll 1$, where $R$ is an appropriate reduced Reynolds number defined by

$$
R=\frac{\epsilon^{3} \tau}{\delta \rho g^{2} \cos ^{2} \alpha}\left(\frac{\tau}{\mu_{0}}\right)^{\frac{2}{N}}
$$

equation (1) leads to

$$
\begin{gathered}
u_{x}+v_{y}+w_{z}=0, \\
\left(\mu u_{z}\right)_{z}=0, \\
-p_{y}+\left(\mu v_{z}\right)_{z}=0, \\
-p_{z}-S_{g}=0
\end{gathered}
$$

at leading order in $\epsilon$ and $\delta$, where we have introduced the notation $S_{g}=\operatorname{sgn}(\cos \alpha)= \pm 1$ (so that $S_{g}=1$ and $S_{g}=-1$ correspond to the sessile and pendent cases, respectively). In particular, the down-slope pressure gradient $p_{x}$ and the down-slope component of gravity are negligible provided that $\epsilon^{2} \ll 1$ and $\epsilon|\tan \alpha| \ll \delta$, respectively; in that case the draining down the plane caused by gravity is negligible in comparison with the flow down the plane driven by the prescribed surface shear stress (though the pressure gradient that arises due to the $z$ component of gravity induces a nonzero lateral flow).

Equations (11)-(14) are to be integrated subject to the boundary conditions (3) at $z=0$ and the leading order balances of normal and tangential stresses at $z=h$ obtained from equation (4), namely

$$
p=-\mathrm{Ca}^{-1} h_{y y}, \quad \mu u_{z}=1, \quad \mu v_{z}=0
$$


where $\mathrm{Ca}=\rho g|\cos \alpha| \epsilon^{2} \ell^{2} / \gamma$ is an appropriate non-dimensional capillary number. Provided that $\mathrm{Ca}^{-1} \ll 1$ the effect of surface tension is negligible, and in that case the solution is simply

$$
\begin{gathered}
p=S_{g}(h-z), \\
u=z, \quad u_{z}=1, \quad \mu=1, \\
v=-\frac{S_{g} h_{y}}{2} z(2 h-z), \\
w=\frac{S_{g}}{6}\left[h_{y} z^{2}(3 h-z)\right]_{y} .
\end{gathered}
$$

Substituting (17) and (18) into equation (7) gives

$$
\bar{u}=\frac{1}{2} h^{2}, \quad \bar{v}=-\frac{S_{g}}{3} h^{3} h_{y}
$$

and then the kinematic condition (5) yields the governing partial differential equation for $h$, namely

$$
3 h_{t}=S_{g}\left(h^{3} h_{y}\right)_{y}-3 h h_{x}
$$

which, we note, is independent of the power-law index $N$. From (20) we have $\bar{u}=0$ at $y= \pm a$, so that the zero-mass-flux condition (6) at the contact lines reduces to $\bar{v}=0$ at $y= \pm a$; thus we have the contact-line conditions

$$
h=0 \quad \text { at } \quad y= \pm a, \quad h^{3} h_{y} \rightarrow 0 \quad \text { as } \quad y \rightarrow \pm a
$$

\section{A similarity solution}

Equation (21) has an unsteady similarity solution of the form

$$
h=\left|\frac{x}{t}\right| H(\eta), \quad \eta=\frac{\sqrt{3}|t|}{|x|^{\frac{3}{2}}} y,
$$

where the function $H=H(\eta)(\geq 0)$ of the independent variable $\eta$ is to be determined, and where

the numerical factor of $\sqrt{3}$ has been included in the definition of $\eta$ for subsequent convenience. With (23), equation (21) reduces to the ordinary differential equation

$$
S_{t}\left(\eta H^{\prime}-H\right)=S_{g}\left(H^{3} H^{\prime}\right)^{\prime}+S_{x}\left(\frac{3}{4} \eta\left(H^{2}\right)^{\prime}-H^{2}\right)
$$


for $H$, where a dash denotes differentiation with respect to $\eta$, and we have introduced the notation $S_{t}=\operatorname{sgn}(t)= \pm 1$ and $S_{x}=\operatorname{sgn}(x)= \pm 1$. The cases $S_{t}=1$ and $S_{t}=-1$ correspond to time running from 0 to $\infty$ and from $-\infty$ to 0 , respectively, and the cases $S_{x}=1$ and $S_{x}=-1$ correspond to the fluid being in $x>0$ and $x<0$, respectively. Thus in the differential equation (24) there are four distinct cases to consider, namely $S_{t}=S_{g}=S_{x}, S_{t}=-S_{g}=S_{x}$, $S_{t}=S_{g}=-S_{x}$ and $S_{t}=-S_{g}=-S_{x}$. Furthermore, each of these cases has two different physical interpretations; for instance, physically the case $S_{t}=-S_{g}=S_{x}=1$ corresponds to a pendent film in $x>0$, with $t>0$, whereas the case $S_{t}=-S_{g}=S_{x}=-1$ corresponds to a sessile film in $x<0$, with $t<0$, but the two cases will have exactly the same mathematical solution. We note that, like (21), equation (24) is independent of $N$.

We now investigate solutions of the form (23) representing a rivulet (in Section 4) and a dry patch (in Section 5).

\section{A rivulet}

For a symmetric rivulet, appropriate boundary and symmetry conditions on $H$ are

$$
H=H_{0}, \quad H^{\prime}=0 \quad \text { at } \quad \eta=0
$$

where $H_{0} \geq 0$ is to be determined; the (unknown) position of the contact line is denoted by $\eta=\eta_{0}$, so that the fluid occupies $|\eta| \leq \eta_{0}$, and from (22) we have

$$
H=0 \quad \text { at } \quad \eta=\eta_{0}, \quad H^{3} H^{\prime} \rightarrow 0 \quad \text { as } \quad \eta \rightarrow \eta_{0}
$$

From (23) the middle thickness of the rivulet, $h_{\mathrm{m}}=h(x, 0, t)$, and the semi-width of the rivulet vary with $x$ and $t$ according to

$$
h_{\mathrm{m}}=\left|\frac{x}{t}\right| H_{0}, \quad a=\frac{|x|^{\frac{3}{2}}}{\sqrt{3}|t|} \eta_{0},
$$

predicting that at any time $t$ the rivulet widens or narrows according to $|x|^{3 / 2}$ and thickens or thins according to $|x|$, and that at any station $x$ the rivulet widens or narrows according to $|t|^{-1}$ and thickens or thins according to $|t|^{-1}$, independent of the power-law index $N$. The rivulet 
has a "nose" that, according to the similarity solution, remains stationary at the origin $O$ for all time. We note that the assumptions that the rivulet is thin and slender are not violated at the nose, since $\partial a / \partial x=0$ and $\partial h_{\mathrm{m}} / \partial x$ is finite there; however, the fact that there is a cusp at the nose means that surface-tension effects (neglected in the present analysis) will inevitably be significant there.

Of the four cases $S_{t}=S_{g}=S_{x}, S_{t}=-S_{g}=S_{x}, S_{t}=S_{g}=-S_{x}$ and $S_{t}=-S_{g}=-S_{x}$ to consider in the differential equation (24) we shall show shortly that for a rivulet only the case $S_{t}=-S_{g}=S_{x}$ can lead to physically realisable solutions.

The cross-sectional area of the rivulet at any station $x$, denoted by $A$ and scaled with $\epsilon^{2} \delta \ell^{2}$, is given by

$$
A=2 \int_{0}^{a} h \mathrm{~d} y=\frac{I_{1}|x|^{\frac{5}{2}}}{\sqrt{3}|t|^{2}},
$$

and the total volume flux of fluid across any section $x=$ constant, denoted by $Q$ and scaled with $\epsilon^{2} \delta \ell^{2} U$, is given by

$$
Q=2 \int_{0}^{a} \bar{u} \mathrm{~d} y=\frac{I_{2}|x|^{\frac{7}{2}}}{2 \sqrt{3}|t|^{3}},
$$

where $I_{n}$ is defined as

$$
I_{n}=2 \int_{0}^{\eta_{0}} H^{n} \mathrm{~d} \eta .
$$

Also the area of a finite (non-dimensional) length $L_{0}$ of the wetted part of the inclined plane in $0 \leq|x| \leq L_{0}$ (that is, the "footprint" of that part of the rivulet), denoted by $A_{0}$ and scaled with $\epsilon \ell^{2}$, is given by

$$
A_{0}=2 \int_{0}^{L_{0}} a \mathrm{~d} x=\frac{4 L_{0}^{\frac{5}{2}} \eta_{0}}{5 \sqrt{3}|t|} .
$$

Near $\eta=0$ we find straightforwardly from (24) and (25) that $H$ satisfies

$$
H=H_{0}+\frac{S_{x} H_{0}-S_{t}}{2 S_{g} H_{0}^{2}} \eta^{2}+O\left(\eta^{4}\right)
$$

when $H_{0} \neq 0$. Near the contact line $\eta=\eta_{0}$ there are two possible forms for $H$ satisfying the differential equation (24) and boundary condition $(26)_{1}$, namely

$$
H \sim\left[-\frac{3 S_{t} \eta_{0}}{S_{g}}\left(\eta_{0}-\eta\right)\right]^{\frac{1}{3}}
$$


(valid only provided that $S_{t}=-S_{g}$ ) and

$$
H \sim C\left(\eta_{0}-\eta\right)^{\frac{1}{4}}-\frac{4 S_{t} \eta_{0}}{5 S_{g} C^{2}}\left(\eta_{0}-\eta\right)^{\frac{1}{2}}
$$

(valid for any $S_{t}= \pm 1$ and $S_{g}= \pm 1$ ), where $C$ is a positive constant. Note that both (33) and (34) have $H^{\prime} \rightarrow \infty$ as $\eta \rightarrow \eta_{0}$, showing that the lubrication approximation always fails near the contact lines (and that there is no freedom to impose prescribed conditions on the threephase contact angles). However, with (34) the zero-mass-flux condition $(26)_{2}$ would require that $C=0$; therefore the behaviour (34) is immediately eliminated, so that the only possible behaviour near $\eta=\eta_{0}$ is given by (33). Furthermore, the two cases with $S_{t}=S_{g}$, in which (34) is the only possible behaviour near the contact line, are also eliminated, and so we are left with only the cases with $S_{t}=-S_{g}$; determining the physical solutions for these cases from the condition $C=0$ will be discussed shortly.

Since the rivulet has two contact lines, at $\eta= \pm \eta_{0}$, the function $H(\geq 0)$ must have at least one maximum in $|\eta| \leq \eta_{0}$. From equation (24), at any stationary point of the free surface (where $H^{\prime}=0$ ) for the cases with $S_{t}=-S_{g}$ we have

$$
H^{2} H^{\prime \prime}=1-S_{t} S_{x} H
$$

provided that $H \neq 0$. In the case $S_{t}=-S_{g}=-S_{x}$, equation (35) shows immediately that any stationary point of $H$ is a minimum, so there can be no solution for $H$ with a maximum, and therefore this case is eliminated.

With all other cases eliminated, the only case that could lead to physically realisable solutions is $S_{t}=-S_{g}=S_{x}$. The sessile case $S_{t}=-S_{g}=S_{x}=-1$ corresponds to fluid in $x<0$ flowing in the direction of $\tau$ and "piling up" at $x=0$, notionally becoming infinitely deep at $t=0$, the substrate in $x>0$ remaining unwetted. The pendent case $S_{t}=-S_{g}=S_{x}=1$ corresponds to a deep (notionally infinitely deep) layer of fluid in $x>0$ at $t=0$ collapsing in the direction of $\tau$ as time elapses, the substrate in $x<0$ remaining unwetted. Of course, in both cases, by a simple shift of origin the position $x=0$ may be replaced by any fixed finite position $x=x_{0}$ without affecting the form of solution; similarly, by a simple shift in time the instant $t=0$ at which the solution is singular may instead be taken to be any other fixed instant $t=t_{0}$. 
With $S_{t}=-S_{g}=S_{x}$, equation (24) becomes

$$
\left(H^{3} H^{\prime}\right)^{\prime}-\frac{3}{4} \eta\left(H^{2}\right)^{\prime}+\eta H^{\prime}+H^{2}-H=0
$$

A closed-form solution of this differential equation is not available, and so it must, in general, be solved numerically. We did this in two stages, first by using a shooting method to solve (36) subject to $(25)$ to determine the value of $\eta_{0}$ in $(26)_{1}$ for a given value of $H_{0}$, and then by using the condition $C=0$ to select the physically realisable solutions from this family of solutions; $H_{0}$ and $\eta_{0}$ are thus determined as part of the solution.

The results for $\eta_{0}$ as a function of $H_{0}$ obtained by shooting are shown in Figure 3 . This function has four branches, labelled as branches 1, 2, 3 and 4 in Figure 3; branch 1 extends from $H_{0} \simeq 0.0000$ to $H_{0} \simeq 0.9250$, branch 2 from $H_{0} \simeq 0.9250$ to $H_{0} \simeq 1.0000$, branch 3 from $H_{0} \simeq 1.0000$ to $H_{0} \simeq 1.2708$, and branch 4 from $H_{0} \simeq 1.2708$ to large values of $H_{0}$, there being asymptotes to the function at $H_{0} \simeq 0.9250, H_{0} \simeq 1.0000$ and $H_{0} \simeq 1.2708$. (Here and subsequently, quantities obtained numerically are given to four decimal places.) There is a solution $\eta_{0}$ for every value of $H_{0}$ except at the asymptotes, at which there is no solution. Figure 4 shows numerically calculated cross-sectional profiles $H$ for a range of values of $H_{0}$ on the different branches. Equation (35) shows that any stationary point for which $H<1$ is a minimum, and any stationary point for which $H>1$ is a maximum. Solutions on branches 1 and 2 are "double-humped", with a minimum at $\eta=0$ and two equal maxima symmetrically placed about $\eta=0$, and solutions on branch 3 are "triple-humped", with a maximum at $\eta=0$, and two equal maxima and two equal minima symmetrically placed about $\eta=0$; solutions on branch 4 are "single-humped", with a single maximum at $\eta=0$.

We can understand the large- $H_{0}$ limit shown in Figure 3 by analysing the behaviour of $H$ and $\eta_{0}$ in the limit $H_{0} \rightarrow \infty$. From the numerically calculated solutions, it is found that $H=O\left(H_{0}\right)$ and $\eta_{0}=O\left(H_{0}\right)$, and so we write

$$
H=H_{0} \bar{H}(\bar{\eta}), \quad \eta=H_{0} \bar{\eta}, \quad \eta_{0}=H_{0} \bar{\eta}_{0}
$$

then at leading order equation (36) reduces to

$$
\left(\bar{H}^{3} \bar{H}^{\prime}\right)^{\prime}-\frac{3}{4} \bar{\eta}\left(\bar{H}^{2}\right)^{\prime}+\bar{H}^{2}=0
$$


which is readily solved numerically subject to the boundary conditions

$$
\bar{H}(0)=1, \quad \bar{H}^{\prime}(0)=0,
$$

to yield $\bar{\eta}_{0} \simeq 0.6801$. Figure 5 shows comparisons between profiles $H$ for $H_{0}=3$ and $H_{0}=10$ computed numerically (full curves) and from the leading order asymptotic solution in the limit $H_{0} \rightarrow \infty$ (dashed curves), demonstrating the good agreement between the two for sufficiently large $H_{0}$. In addition, Figure 3 includes (as a dashed-dotted curve) the leading order asymptotic solution for $\eta_{0}$ as a function of $H_{0}$ in the limit $H_{0} \rightarrow \infty$.

Next we identify the physically realisable solutions from the family of solutions shown in Figure 3. In order to do this, we must impose condition $(26)_{2}$, or equivalently the condition $C=0$ in (34). A simple and accurate way to determine $C$ is to integrate equation (36) from $\eta=0$ to $\eta=\eta_{0}$, which leads to

$$
\int_{0}^{\eta_{0}} 7 H^{2}-8 H \mathrm{~d} \eta= \begin{cases}0 & \text { for }(33) \\ C^{4} & \text { for }(34)\end{cases}
$$

so that $C$ in (34) is given by

$$
C=\left[\int_{0}^{\eta_{0}} 7 H^{2}-8 H \mathrm{~d} \eta\right]^{\frac{1}{4}}=\left[\frac{1}{2}\left(7 I_{2}-8 I_{1}\right)\right]^{\frac{1}{4}}
$$

which is evaluated with the numerical solution for $H(\eta)$. Also these values of $C$ were checked against values that we obtained from (34) using the numerical solution near $\eta=\eta_{0}$. Figure 6 shows a plot of $C$ calculated from (41) as a function of $H_{0}$. From this plot we see that there is only one value of $H_{0}$ for which $C=0$, namely $H_{0} \simeq 1.2708$, for which the corresponding value of $\eta_{0}$ is $\eta_{0} \simeq 1.9152$. Thus our main conclusion is that there is a unique physically realisable solution of the type sought for an unsteady rivulet. This solution has a single-humped crosssectional profile; the associated values of $I_{1}$ and $I_{2}$ in the expressions (28) and (29) for the cross-sectional area $A$ and flux $Q$ are given by equation (30) as $I_{1} \simeq 4.2495$ and $I_{2} \simeq 4.8565$, consistent with the fact that $8 I_{1}=7 I_{2}$ in the case $C=0$.

As mentioned previously, although the cases where $S_{t}=-S_{g}=S_{x}=1$ and $S_{t}=-S_{g}=$ $S_{x}=-1$ have the same mathematical solution, the two cases have different physical interpretations, the former representing a pendent rivulet and the latter a sessile rivulet. However, 
numerical results of a stability study of corresponding similarity solutions for a gravity-driven flow of a Newtonian fluid by Yatim et al. [44] suggest that the sessile case is stable but that the pendent case is unstable, indicating that we may also tentatively eliminate the possibility of a pendent rivulet for the present problem. Figure 7 shows a three-dimensional plot of the free surface $z=h$ of the sessile rivulet predicted by the similarity solution (23) for the physical solution $H_{0} \simeq 1.2708, \eta_{0} \simeq 1.9152$, at times $t=-5,-2$ and -1 ; the insert shows the (single-humped) cross-sectional profile of the solution. In particular, Figure 7 illustrates that the sessile rivulet becomes narrower and thinner with $|x|$ as it flows down the plane, but becomes wider and thicker as time elapses (from $t \rightarrow-\infty$ to $t=0$ ), eventually exhibiting a finite-time singularity, becoming infinite everywhere at $t=0$.

So far we have interpreted the similarity solution (23) as representing an infinitely long rivulet of infinite volume. However, in the spirit of Huppert's [42] analysis of two-dimensional gravity-driven unsteady flow of a thin film down an inclined plane, we may alternatively interpret the solution as representing a rivulet of (prescribed) finite volume, by truncating it at some finite length $L=L(t)$ (scaled with $\ell$ ), so that in the sessile case it occupies $-L \leq x \leq 0$. The volume of fluid in such a rivulet, denoted by $V$ and scaled with $\epsilon^{2} \delta \ell^{3}$, is given by

$$
V=\int_{-L(t)}^{0} \int_{-a}^{a} h \mathrm{~d} y \mathrm{~d} x=\frac{2 I_{1} L^{\frac{7}{2}}}{7 \sqrt{3} t^{2}}
$$

and so the length $L$ of the rivulet at time $t$ is given by

$$
L=\left(\frac{7 \sqrt{3} V t^{2}}{2 I_{1}}\right)^{\frac{2}{7}}
$$

decreasing with $t$ according to $t^{4 / 7}$.

\section{$5 \quad$ A dry patch}

The approach discussed in Section 4 may also be used to investigate unsteady shear-stressdriven flow of an infinitely wide thin film of a Newtonian or a non-Newtonian power-law fluid around a symmetric slender dry patch on an inclined plane. Conditions for the dry patch to be slender, for the film to be thin, and for the gravity-driven draining to be negligible in 
comparison with the flow driven by $\tau$ are given by $\epsilon \ll 1, \delta \ll 1$ and $\epsilon|\tan \alpha| \ll \delta$, respectively. In that case the governing equation (21) again holds, but now the fluid occupies $|y| \geq a$, where $a=a(x)$ denotes the semi-width of the dry patch, the region $|y|<a$ of the inclined plane being dry. Seeking a similarity solution of the form (23) we find that $H(\eta)$ again satisfies the ordinary differential equation (24), now valid in $|\eta| \geq \eta_{0}$, where $\eta=\eta_{0}$ again denotes the (unknown) position of the contact line where $H=0$ (and the fluid region now corresponds to $|\eta| \geq \eta_{0}$ ). The similarity solution predicts that at any time $t$ the dry patch (whose semi-width $a$ is as in (27)) widens or narrows according to $|x|^{3 / 2}$ and the film thickens or thins according to $|x|$, and that at any station $x$ the dry patch widens or narrows like $|t|^{-1}$ and the film thickens or thins like $|t|^{-1}$, independent of the power-law index $N$.

There are again four distinct cases to consider in equation (24), namely $S_{t}=S_{g}=S_{x}$, $S_{t}=-S_{g}=S_{x}, S_{t}=S_{g}=-S_{x}$ and $S_{t}=-S_{g}=-S_{x}$, each of which again has two different physical interpretations, namely the sessile case and the pendent case. We look for solutions $H$ satisfying $H \rightarrow H_{\infty}$ as $\eta \rightarrow \infty$, where $H_{\infty}$ is a positive constant representing the depth of the film far from the dry patch; then equation (24) immediately gives $S_{t} H_{\infty}=S_{x} H_{\infty}^{2}$, which requires both that $S_{t}=S_{x}$ and that $H_{\infty}=1$. Therefore appropriate far-field conditions are

$$
H \rightarrow 1, \quad H^{\prime} \rightarrow 0 \quad \text { as } \quad \eta \rightarrow \infty
$$

(so that the film thickness satisfies $h \rightarrow|x / t|$ as $y \rightarrow \pm \infty$, showing that the film becomes uniform in $y$ far from the dry patch, but varies with $x$ and $t$ ). From equation (44) $H$ satisfies $H \sim 1+F$ with $F \rightarrow 0$ as $\eta \rightarrow \infty$, and so by (24) with $S_{t}=S_{x}$ we find that $F=F(\eta)$ satisfies

$$
S_{g} F^{\prime \prime}+S_{t}\left(\frac{\eta}{2} F^{\prime}-F\right)=0
$$

at leading order as $\eta \rightarrow \infty$. If $S_{t}=S_{g}$ then equation (45) has general solution

$$
F=c_{1}\left(1+\frac{\eta^{2}}{2}\right)+c_{2} \exp \left(-\frac{\eta^{2}}{4}\right) \mathrm{H}_{-3}\left(\frac{\eta}{2}\right)
$$

where $c_{1}$ and $c_{2}$ are constants, and $\mathrm{H}_{-3}$ denotes a Hermite function of degree -3 (see [46]); then equation (44) requires that $c_{1}=0$, and therefore

$$
H \sim 1+c_{2} \eta^{-3} \exp \left(-\frac{\eta^{2}}{4}\right) \quad \text { as } \quad \eta \rightarrow \infty
$$


showing that $H$ approaches its far-field value 1 monotonically as $\eta \rightarrow \infty$. On the other hand if $S_{t}=-S_{g}$ then no solution of (45) satisfying $F \rightarrow 0$ as $\eta \rightarrow \infty$ is possible; therefore only the case $S_{t}=S_{g}=S_{x}$ could possibly lead to a physically realisable dry-patch solution of the type sought. The sessile case $S_{t}=S_{x}=S_{g}=1$ corresponds to a layer of fluid in $x>0$ collapsing (in the direction of $\tau$ ) around a contracting dry patch as time elapses, the substrate in $x<0$ remaining unwetted. The pendent case $S_{t}=S_{x}=S_{g}=-1$ corresponds to fluid in $x<0$ flowing (in the direction of $\tau$ ) around an expanding dry patch as time elapses, and "piling up" at $x=0$, the substrate in $x>0$ remaining unwetted.

The free-surface profile $H$ must again satisfy the contact-line conditions in (26). Near the contact line $\eta=\eta_{0}$, the asymptotic behaviour of solutions of (24) given in (33) also holds for a dry patch (now with $S_{t}=S_{g}$ and $\eta \geq \eta_{0}$ ), but (34) is replaced by

$$
H \sim C\left(\eta-\eta_{0}\right)^{\frac{1}{4}}+\frac{4 S_{t} \eta_{0}}{5 S_{g} C^{2}}\left(\eta-\eta_{0}\right)^{\frac{1}{2}}
$$

as $\eta \rightarrow \eta_{0}^{+}$, where $C$ again denotes a positive constant. However, equation (26) again requires that $C=0$, implying that only the case $S_{t}=S_{g}=S_{x}$ could lead to a physically realisable solution, in which case equation (24) becomes

$$
\left(H^{3} H^{\prime}\right)^{\prime}+\frac{3}{4} \eta\left(H^{2}\right)^{\prime}-\eta H^{\prime}-H^{2}+H=0
$$

From equation (49), at any stationary point of the free surface we have

$$
H^{\prime \prime}=\frac{H-1}{H^{2}}
$$

showing that any stationary point with $H<1(H>1)$ would be a maximum (minimum); we conclude that $H$ must increase monotonically from $H=0$ at $\eta=\eta_{0}$ to $H=1$ as $\eta \rightarrow \infty$, with $0 \leq H<1$.

As in the corresponding analysis for a rivulet described previously, we solved the ordinary differential equation (49) numerically by means of a shooting method. For a dry patch we may shoot either from some far-field position $\eta=\eta_{\infty} \gg 1$ (using approximate boundary conditions obtained from (47)) until the solution achieves a contact line at some position $\eta=\eta_{0}$, or from a chosen position $\eta=\eta_{0}$ to see whether the solution settles down to a constant value $H=1$ 
as $\eta$ becomes large; we chose to use the latter method. The numerical computation cannot be started exactly at the contact line $\eta=\eta_{0}$ (because of the singularity there), so instead it was started from a position $\eta=\eta_{0}+\delta$, where $\delta(>0)$ is small; thus in practice we solved equation (49) subject to approximated boundary conditions obtained from (33), namely

$$
H\left(\eta_{0}+\delta\right) \sim\left(3 \eta_{0} \delta\right)^{\frac{1}{3}}, \quad H^{\prime}\left(\eta_{0}+\delta\right) \sim\left(\frac{\eta_{0}}{9 \delta^{2}}\right)^{\frac{1}{3}}
$$

and the computation was then repeated with smaller values of $\delta$ (as small as $\delta=10^{-20}$ ) until the solution converged to within a given tolerance. This procedure was then repeated with different values of $\eta_{0}$. In this way we arrive at our main conclusion, namely that there is only one physically realisable dry-patch solution of the type sought, and that for this solution $\eta_{0} \simeq 0.9573$

As in the rivulet case, it may again be anticipated that the sessile solution will be stable whereas the pendent solution will be unstable. Figure 8 shows a three-dimensional plot of the solution for a sessile film $\left(S_{t}=S_{g}=S_{x}=+1\right)$ at the instant $t=1$; the insert shows the cross-sectional profile of the solution. In particular, Figure 8 illustrates the widening of the dry patch and the thickening of the film with $x$; also we note that, as for the rivulet, the dry patch has a "nose" that remains stationary at $O$ for all time. Figure 9 shows cross-sections at position $x=5$ of the sessile film at times $t=1,2,3,4,5$; in particular, Figure 9 illustrates the narrowing of the dry patch and the thinning of the film with $t$.

The film is of infinite lateral extent, and its cross-sectional area (at constant $x$ ) is infinite, as is the volume flux of fluid down the inclined plane. However, the difference between the cross-sectional area of a film of thickness $|x / t|$ (uniform in $y$ ) and that of a film containing a dry patch with the same thickness far from the patch, denoted $\triangle A$ and scaled with $\epsilon^{2} \delta \ell^{2}$, is finite and is given by

$$
\triangle A=\frac{|x|^{\frac{5}{2}}}{\sqrt{3} t^{2}}\left(\eta_{0}+J_{1}\right),
$$

and similarly the difference in volume flux in the two films, denoted $\triangle Q$ and scaled with $\epsilon^{2} \delta \ell^{2} U$, is finite and is given by

$$
\triangle Q=\frac{|x|^{\frac{7}{2}}}{2 \sqrt{3}|t|^{3}}\left(\eta_{0}+J_{2}\right)
$$


where we have defined the constants $J_{n}$ by

$$
J_{n}=\int_{\eta_{0}}^{\infty} 1-H^{n} \mathrm{~d} \eta,
$$

so that $J_{1} \simeq 0.2382$ and $J_{2} \simeq 0.4090$. (Integration of (49) from $\eta=\eta_{0}$ to $\eta=\infty$ leads to $7 J_{2}-8 J_{1}=\eta_{0}$, numerical confirmation of which provides a check on the accuracy of the numerical solution.) Also the area $A_{0}$ of a finite length $L_{0}$ of the dry part of the inclined plane in $0<|x|<L_{0}$ is again given by (31).

\section{Discussion}

We have obtained similarity solutions of the form (23) representing unsteady flow of a thin slender rivulet of either a Newtonian fluid or a non-Newtonian power-law fluid on an inclined plane, and unsteady flow of a thin film of such a fluid around a slender dry patch on an inclined plane, the flow in each case being driven by a constant longitudinal shear stress applied at the free surface. There were four cases to consider for each problem, but we showed that the only cases that could lead to physically realisable solutions are $S_{t}=-S_{g}=S_{x}$ for a rivulet (representing both a pendent rivulet $\left(S_{g}=-1\right)$ in $x>0$, with $t>0$, contracting with $t$, and a sessile rivulet $\left(S_{g}=1\right)$ in $x<0$, with $t<0$, expanding with $t$ ), and $S_{t}=S_{g}=S_{x}$ for a dry patch (representing both a pendent film flowing around an expanding dry patch in $x<0$, with $t<0$, and a sessile film flowing around a contracting dry patch in $x>0$, with $t>0$ ). In the case of a rivulet we found that there is only one physically realisable solution, with a single-humped cross-sectional profile of thickness $H_{0} \simeq 1.2708$ and semi-width $\eta_{0} \simeq 1.9152$ (in terms of the similarity variables), and in the case of a dry patch there is again only one physically realisable solution, of semi-width $\eta_{0} \simeq 0.9573$, independent of the power-law index $N$.

The analysis includes the Newtonian case (for which $N=1$ ) as a special case. Although $N$ does not appear in the partial differential equation (21) for $h$, nor in the powers of $x$ and $t$ in the similarity solution (23), nor in the ordinary differential equation (24) for $H$, it nevertheless affects the nature of the solutions, since it appears in the velocity scale $U$ in equation (8), and 
hence in the scaling of the shear rate $q$ and of the shear-rate-dependent viscosity $\mu$. The effect of $N$ on the physical appearance of the solutions may be illustrated by a comparison of the rivulet solution for a power-law fluid with $N \neq 1$ with that for a Newtonian fluid $(N=1)$ of the same density $\rho$, on the same incline $\alpha$, subject to the same driving surface shear stress $\tau$, as follows. With a superposed hat used to denote quantities for the Newtonian fluid, its viscosity $\hat{\mu}$ is independent of $\tau$, whereas the (dimensional) viscosity of the power-law fluid in this flow is $\mu=\mu_{0}\left(\tau / \mu_{0}\right)^{(N-1) / N}$; also the length scales $\ell$ and $\hat{\ell}$ for the two fluids are the same, namely $\ell=\tau / \delta^{2} \rho g|\cos \alpha|=\hat{\ell}$, whereas the velocity scales $U$ and $\hat{U}$ are different, namely $U=\left(\tau / \mu_{0}\right)^{1 / N} \epsilon \delta \ell$ and $\hat{U}=(\tau / \hat{\mu}) \epsilon \delta \ell$. Thus at a given position $x$ and time $t$ we find from (27)-(29), (31) and the scalings (8) that

$$
\frac{h_{\mathrm{m}}}{\hat{h}_{\mathrm{m}}}=\frac{a}{\hat{a}}=\frac{A_{0}}{\hat{A}_{0}}=\frac{\mu}{\hat{\mu}}=\frac{\hat{q}}{q}=\left(\frac{\tau}{\tau_{\mathrm{c}}}\right)^{\frac{N-1}{N}}, \quad \frac{A}{\hat{A}}=\frac{Q}{\hat{Q}}=\left(\frac{\tau}{\tau_{\mathrm{c}}}\right)^{\frac{2(N-1)}{N}}
$$

in dimensional terms, where the critical value of $\tau$, denoted $\tau_{\mathrm{c}}$, is defined by $\tau_{\mathrm{c}}=\left(\hat{\mu}^{N} / \mu_{0}\right)^{1 /(N-1)}$ (and where we have used the fact that $H_{0}$ and $\eta_{0}$ are the same for the two fluids, since (24)-(26) are independent of $N)$. Thus for a shear-thinning fluid $(N<1)$, if, for given values of $\mu_{0}$ and $\hat{\mu}, \tau$ satisfies $\tau<\tau_{\mathrm{c}}$ then the quantities in (55) are greater than 1, whereas if $\tau>\tau_{\mathrm{c}}$ then the quantities in (55) are less than 1. Therefore a rivulet of a shear-thinning fluid subject to a weak shear stress $\left(\tau<\tau_{\mathrm{c}}\right)$ will be wider and thicker than a corresponding rivulet of a Newtonian fluid, whereas such a rivulet subject to a strong shear stress $\left(\tau>\tau_{\mathrm{c}}\right)$ will be narrower and thinner than a corresponding rivulet of a Newtonian fluid; a converse statement holds for a rivulet of a shear-thickening fluid. On the other hand, if, for given values of $\tau$ and $\hat{\mu}, \mu_{0}$ satisfies $\mu_{0}>\mu_{0 \mathrm{c}}\left(\mu_{0}<\mu_{0 \mathrm{c}}\right)$, where the critical value of $\mu_{0}$, denoted $\mu_{0 \mathrm{c}}$, is defined by $\mu_{0 \mathrm{c}}=\hat{\mu}^{N} / \tau^{N-1}$, then a rivulet of a power-law fluid will be wider and thicker (narrower and thinner) than a corresponding rivulet of a Newtonian fluid, independent of whether the power-law fluid is shear thinning or shear thickening. Analogous statements hold for flow around a dry patch.

Both the rivulet and dry patch have a "nose" that remains stationary at $O$ for all time. The solution for a sessile rivulet exhibits a finite-time singularity, becoming infinite everywhere at time $t=0$.

Finally, it is worth mentioning that there are significant qualitative differences between 
shear-stress-driven flow studied herein and gravity-driven flow studied earlier by Yatim et al. [44] in the case of a Newtonian fluid. In gravity-driven rivulet flow there are two physically realisable solutions (one with a double-humped cross-sectional profile and the other with a single-humped cross-sectional profile), whereas in shear-stress-driven flow there is only one physically realisable solution (whose cross-sectional profile is single-humped). Furthermore, there are no dry-patch similarity solutions of the type sought in gravity-driven flow, whereas there is a dry-patch solution in the present shear-stress-driven problem.

\section{Acknowledgement}

The first author (YMY) wishes to thank the Ministry of Higher Education (MOHE), Malaysia and Universiti Sains Malaysia for financial support via an Academic Staff Training Fellowship.

\section{References}

[1] A. C. King and E. O. Tuck, "Thin liquid layers supported by steady air-flow surface traction," J. Fluid Mech. 251, 709-718 (1993).

[2] A. C. King, E. O. Tuck and J.-M. Vanden-Broeck, "Air-blown waves on thin viscous sheets," Phys. Fluids A 5, 973-978 (1993).

[3] M. Villegas-Diaz, H. Power and D. S. Riley, "Analytical and numerical studies of the stability of thin-film rimming flow subject to surface shear," J. Fluid Mech. 541, 317-344 (2005).

[4] H. K. Moffatt, "Behaviour of a viscous film on the outer surface of a rotating cylinder," J. Méc. 16, 651-673 (1977).

[5] S. K. Wilson and B. R. Duffy, "Unidirectional flow of a thin rivulet on a vertical substrate subject to a prescribed uniform shear stress at its free surface," Phys. Fluids 17, 1081051-108105-4 (2005). 
[6] J. M. Sullivan, S. K. Wilson and B. R. Duffy, "A thin rivulet of perfectly wetting fluid subject to a longitudinal surface shear stress," Q. J. Mech. Appl. Math. 61, 25-61 (2008).

[7] I. S. McKinley, S. K. Wilson and B. R. Duffy, "Spin coating and air-jet blowing of thin viscous drops," Phys. Fluids 11, 30-47 (1999).

[8] I. S. McKinley and S. K. Wilson, "The linear stability of a ridge of fluid subject to a jet of air," Phys. Fluids 13, 872-883 (2001).

[9] I. S. McKinley and S. K. Wilson, "The linear stability of a drop of fluid during spin coating or subject to a jet of air," Phys. Fluids 14, 133-142 (2002).

[10] I. Eames, M. A. Gilbertson and M. Landeryou, "The effect of an ambient flow on the spreading of a viscous gravity current," J. Fluid Mech. 523, 261-275 (2005).

[11] L. W. Schwartz, "On the asymptotic analysis of surface-stress-driven thin-layer flow," J. Engng Math. 39, 171-188 (2001).

[12] D. Holland, S. K. Wilson and B. R. Duffy, "Similarity solutions for slender rivulets with thermocapillarity," Q. J. Mech. Appl. Math. 56, 411-439 (2003).

[13] E. Y. Gatapova and O. A. Kabov, "Shear-driven flows of locally heated liquid films," Int. J. Heat Mass Transfer 51, 4797-4810 (2008).

[14] E. B. Dussan V., "On the ability of drops to stick to surfaces of solids. Part 3. The influence of the motion of the surrounding fluid on dislodging drops," J. Fluid Mech. 174, 381-397 (1987).

[15] X. Li and C. Pozrikidis, "Shear flow over a liquid drop adhering to a solid surface," J. Fluid Mech. 307, 167-190 (1996).

[16] P. Dimitrakopoulos and J. J. L. Higdon, "Displacement of fluid droplets from solid surfaces in low-Reynolds-number shear flows," J. Fluid Mech. 336, 351-378 (1997).

[17] P. Dimitrakopoulos, "Deformation of a droplet adhering to a solid surface in shear flow: onset of interfacial sliding," J. Fluid Mech. 580, 451-466 (2007). 
[18] H. Ding and P. D. M. Spelt, "Onset of motion of a three-dimensional droplet on a wall in shear flow at moderate Reynolds number," J. Fluid Mech. 599, 341-362 (2008).

[19] H. Ding, M. N. H. Gilani and P. D. M. Spelt, "Sliding, pinch-off and detachment of a droplet on a wall in shear flow," J. Fluid Mech. 644, 217-244 (2010).

[20] D. E. Hartley and W. Murgatroyd, "Criteria for the break-up of thin liquid layers flowing isothermally over solid surfaces," Int. J. Heat Mass Transfer 7, 1003-1015 (1964).

[21] W. Murgatroyd, "The role of shear and form forces in the stability of a dry patch in two-phase film flow," Int. J. Heat Mass Transfer 8, 297-301 (1965).

[22] D. G. Penn, M. L. de Bertodano, P. S. Lykoudis and S. G. Beus, "Dry patch stability of shear driven liquid films," J. Fluids Eng. 123, 857-862 (2001).

[23] A. B. Ponter, G. A. Davies, T. K. Ross and P. G. Thornley, "The influence of mass transfer on liquid film breakdown," Int. J. Heat Mass Transfer 10, 349-359 (1967).

[24] S. K. Wilson, B. R. Duffy and S. H. Davis, "On a slender dry patch in a liquid film draining under gravity down an inclined plane," Euro. J. Appl. Math. 12, 233-252 (2001).

[25] R. P. Agarwal and D. O’Regan, "Infinite interval problems arising in the model of a slender dry patch in a liquid film draining under gravity down an inclined plane," Methods Appl. Anal. 10, 363-376 (2003).

[26] D. Holland, S. K. Wilson and B. R. Duffy, "Similarity solutions for slender dry patches with thermocapillarity," J. Engng Math. 44, 369-394 (2002).

[27] T. Podgorski, J.-M. Flesselles and L. Limat, "Dry arches within flowing films," Phys. Fluids 11, 845-852 (1999).

[28] T. Podgorski, J.-M. Flesselles and L. Limat, "Curvature of a dry patch boundary in a flowing film," C. R. Acad. Sci., Ser IV: Phys., Astrophys. 2, 1361-1367 (2001).

[29] E. Rio and L. Limat, "Wetting hysteris of a dry patch left inside a flowing film," Phys. Fluids 18, 032102-1-032102-8 (2006). 
[30] S. D. R. Wilson, "The stability of a dry patch on a wetted wall," Int. J. Heat Mass Transfer 17, 1607-1615 (1974).

[31] J. Sébilleau, L. Lebon and L. Limat, "Stability of a dry patch in a viscous flowing fllm,' Eur. Phys. J. Special Topics 166, 139-142 (2009).

[32] J. P. Pascal, "A two-layer model for a non-Newtonian gravity current subjected to wind shear," Acta Mechanica 162, 83-98 (2003).

[33] J. P. Pascal and S. J. D. D'Alessio, "Instability of power-law fluid flows down an incline subjected to wind stress," Appl. Math. Modelling 31, 1229-1248 (2007).

[34] S. K. Wilson, B. R. Duffy and R. Hunt, "A slender rivulet of a power-law fluid driven by either gravity or a constant shear stress at the free surface," Q. J. Mech. Appl. Math. 55, 385-408 (2002).

[35] P. C. Smith, "A similarity solution for slow viscous flow down an inclined plane," J. Fluid Mech. 58, 275-288 (1973).

[36] S. D. R. Wilson and S. L. Burgess, "The steady, spreading flow of a rivulet of mud," J. Non-Newtonian Fluid Mech. 79, 77-85 (1998).

[37] C. A. Perazzo and J. Gratton, "Exact solutions for two-dimensional steady flows of a power-law liquid on an incline," Phys. Fluids 17, 013102-1-013102-8 (2005).

[38] S. H. Smith, "On initial value problems for the flow in a thin sheet of viscous liquid," J. Appl. Math. Phys. 20, 556-560 (1969).

[39] R. E. Pattle, "Diffusion from an instantaneous point source with a concentration-dependent coefficient," Q. J. Mech. Appl. Math. 12, 407-409 (1959).

[40] H. E. Huppert, "The propagation of two-dimensional and axisymmetric viscous gravity currents over a rigid horizontal surface," J. Fluid Mech. 121, 43-58 (1982).

[41] V. A. Gorodtsov, "Spreading of a film of nonlinearly viscous liquid over a horizontal smooth solid surface," J. Engng Phys. 57, 879-884 (1990). 
[42] H. E. Huppert, "Flow and instability of a viscous current down a slope," Nature 300, $427-429$ (1982).

[43] J. R. Lister, "Viscous flows down an inclined plane from point and line sources," J. Fluid Mech. 242, 631-653 (1992).

[44] Y. M. Yatim, B. R. Duffy, S. K. Wilson and R. Hunt, "Similarity solutions for unsteady gravity-driven slender rivulets," Q. J. Mech. Appl. Math. (to appear).

[45] G. W. Young, "Mathematical description of viscous free surface flows," in "Free boundaries in viscous flows", R. A. Brown and S. H. Davis editors, 1-27. (Springer-Verlag 1994).

[46] http://functions.wolfram.com/HypergeometricFunctions/HermiteHGeneral/ 


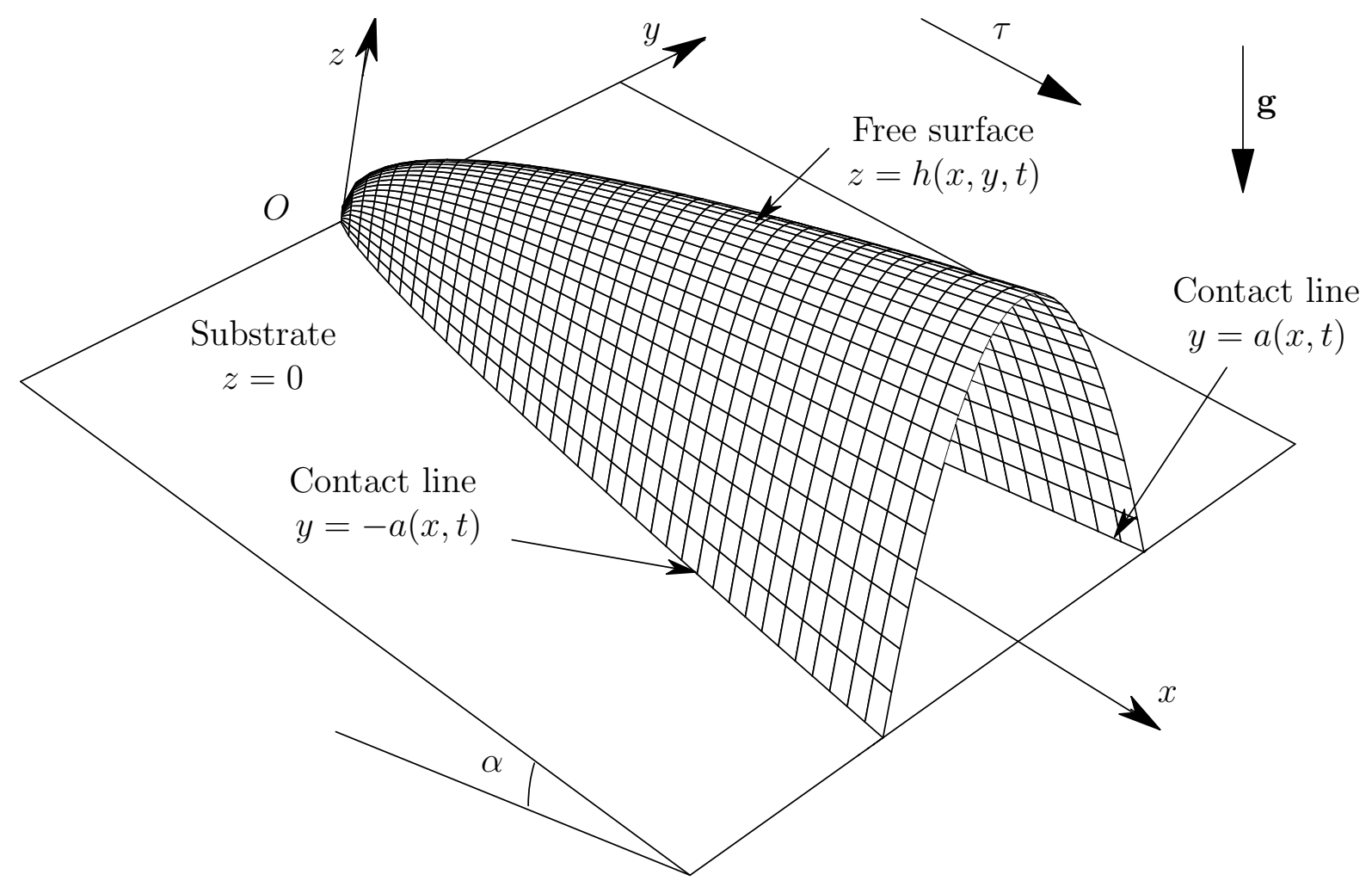

Figure 1: Sketch of the geometry of the rivulet problem. 


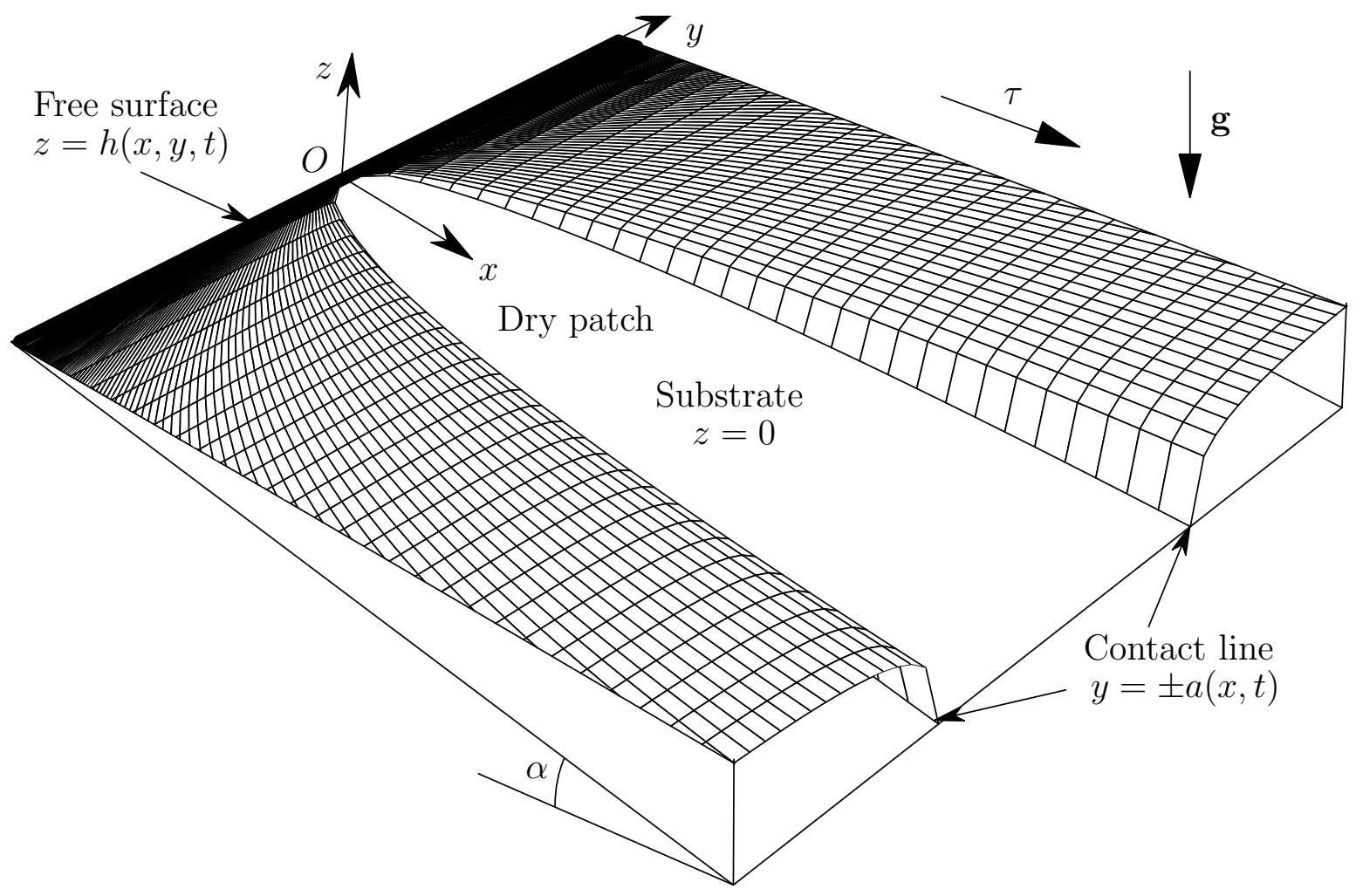

Figure 2: Sketch of the geometry of the dry-patch problem. 


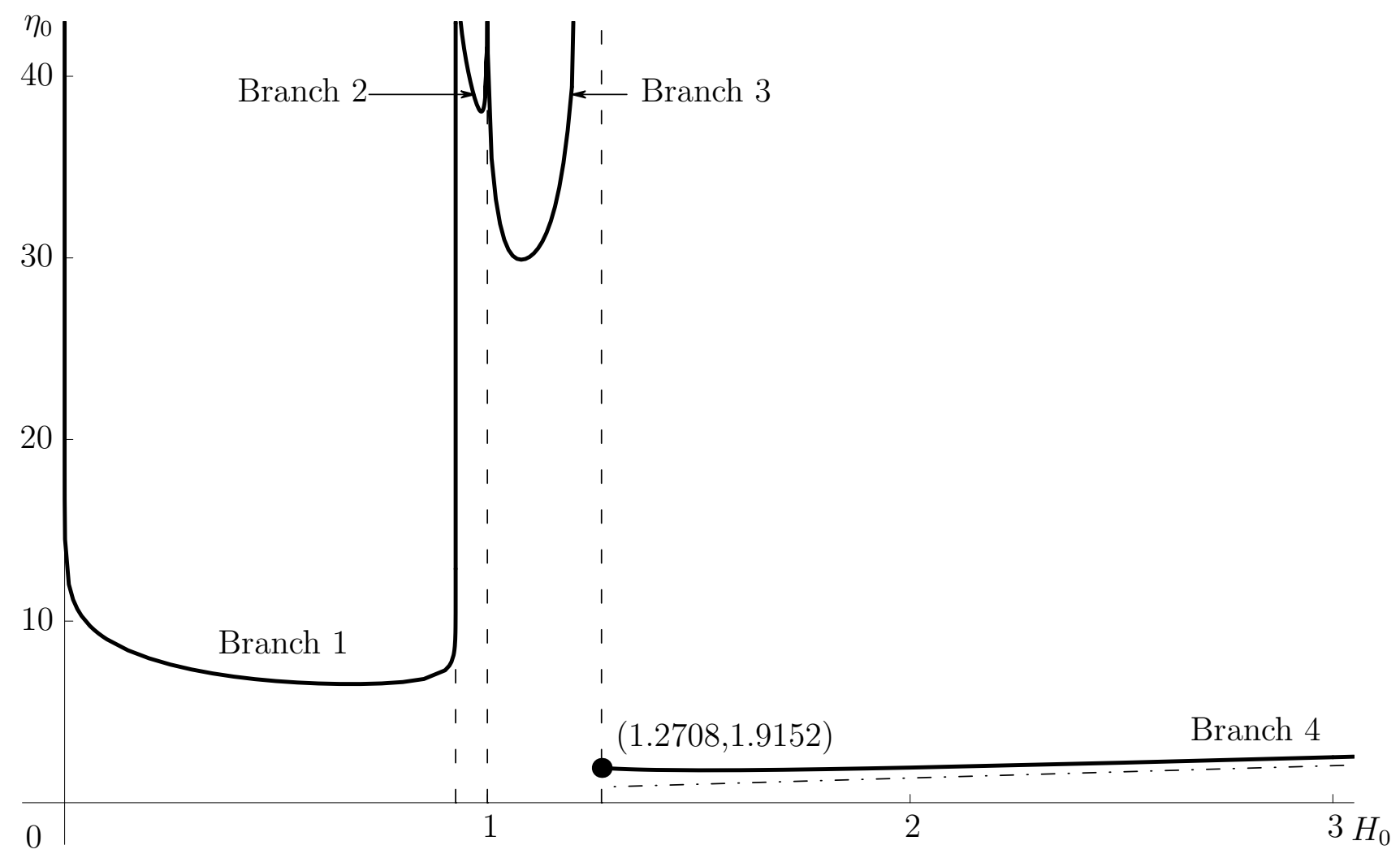

Figure 3: Plot of $\eta_{0}$ as a function of $H_{0}$, obtained by solving (36) numerically subject to (25). The dashed-dotted curve is the leading-order asymptotic solution in the limit $H_{0} \rightarrow \infty$. 


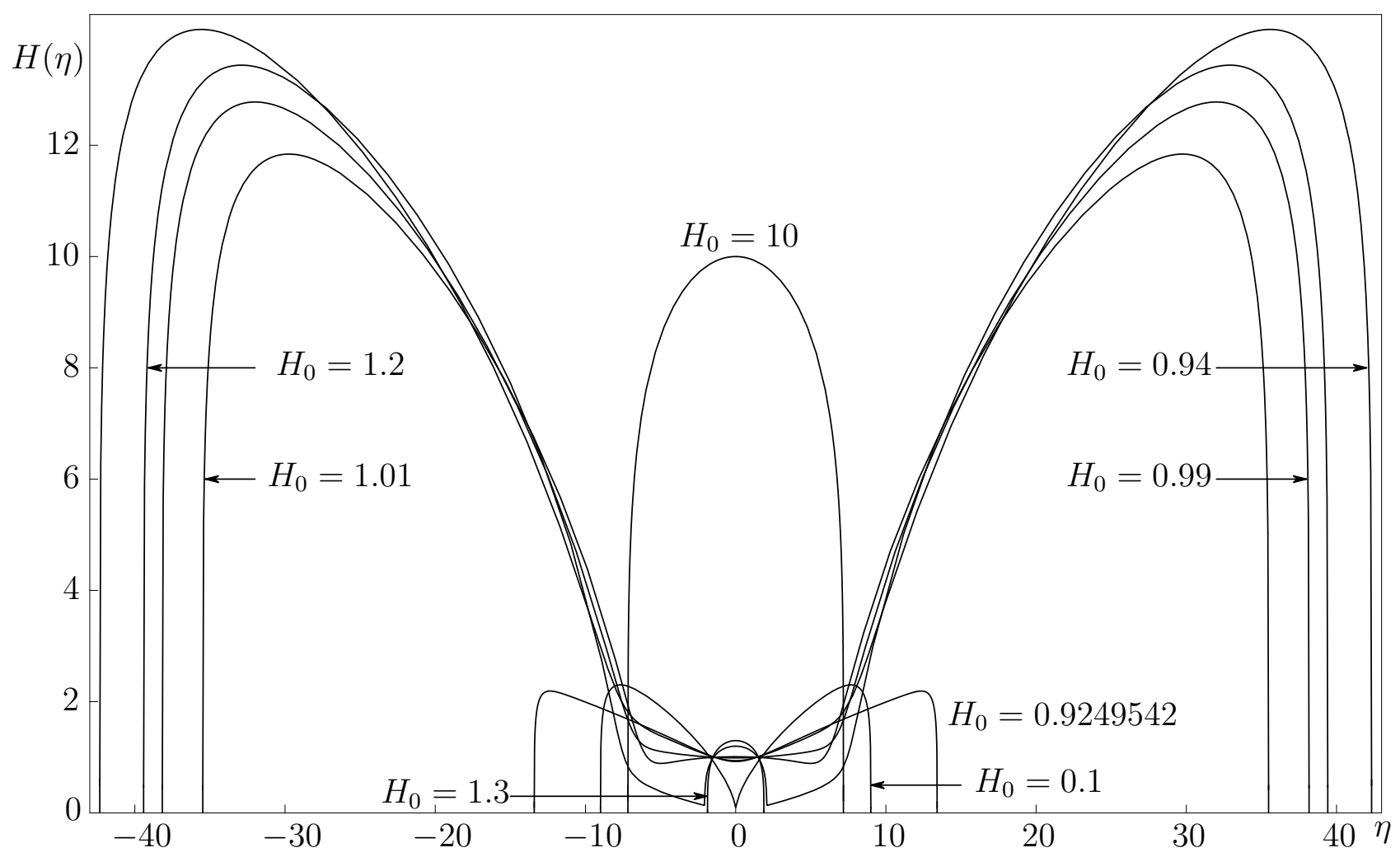

Figure 4: Numerically calculated cross-sectional rivulet profiles $H=H(\eta)$ for $H_{0}=0.1$ and 0.9249542 (branch 1), 0.94 and 0.99 (branch 2), 1.01 and 1.2 (branch 3), and 1.3 and 10 (branch $4)$. 

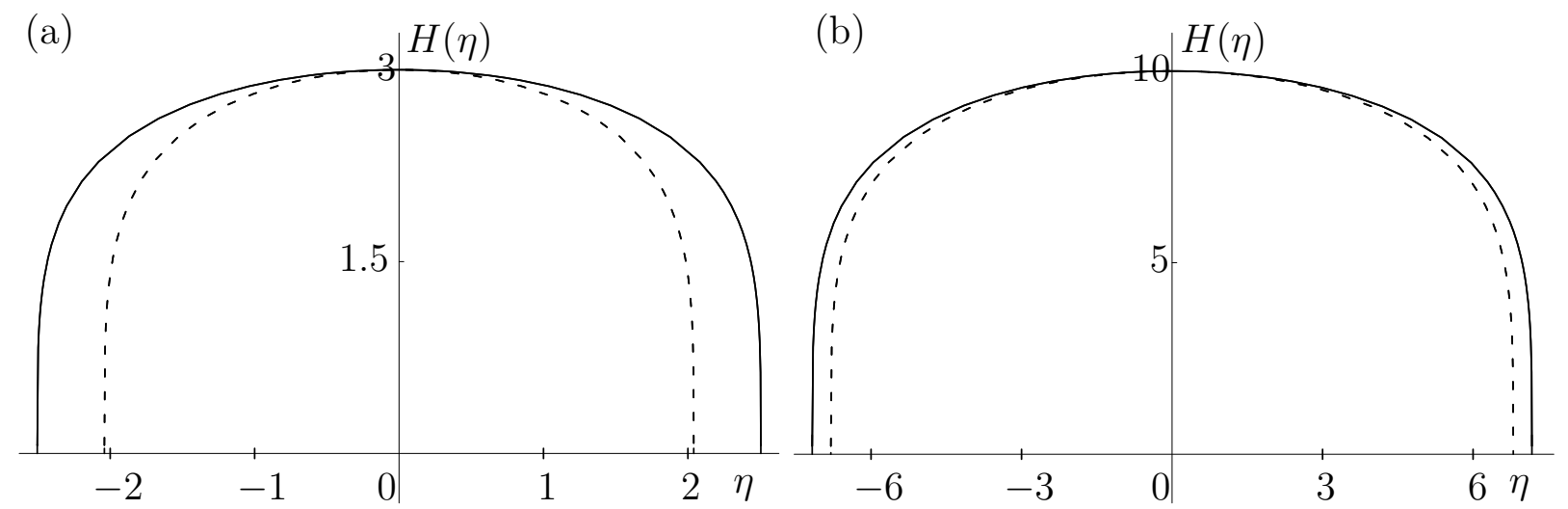

Figure 5: Cross-sectional rivulet profiles $H=H(\eta)$ obtained by solving (36) numerically subject to (25) (full curves) and from the leading order asymptotic solution in the limit $H_{0} \rightarrow \infty$ (dashed curves) for (a) $H_{0}=3$ and (b) $H_{0}=10$. 


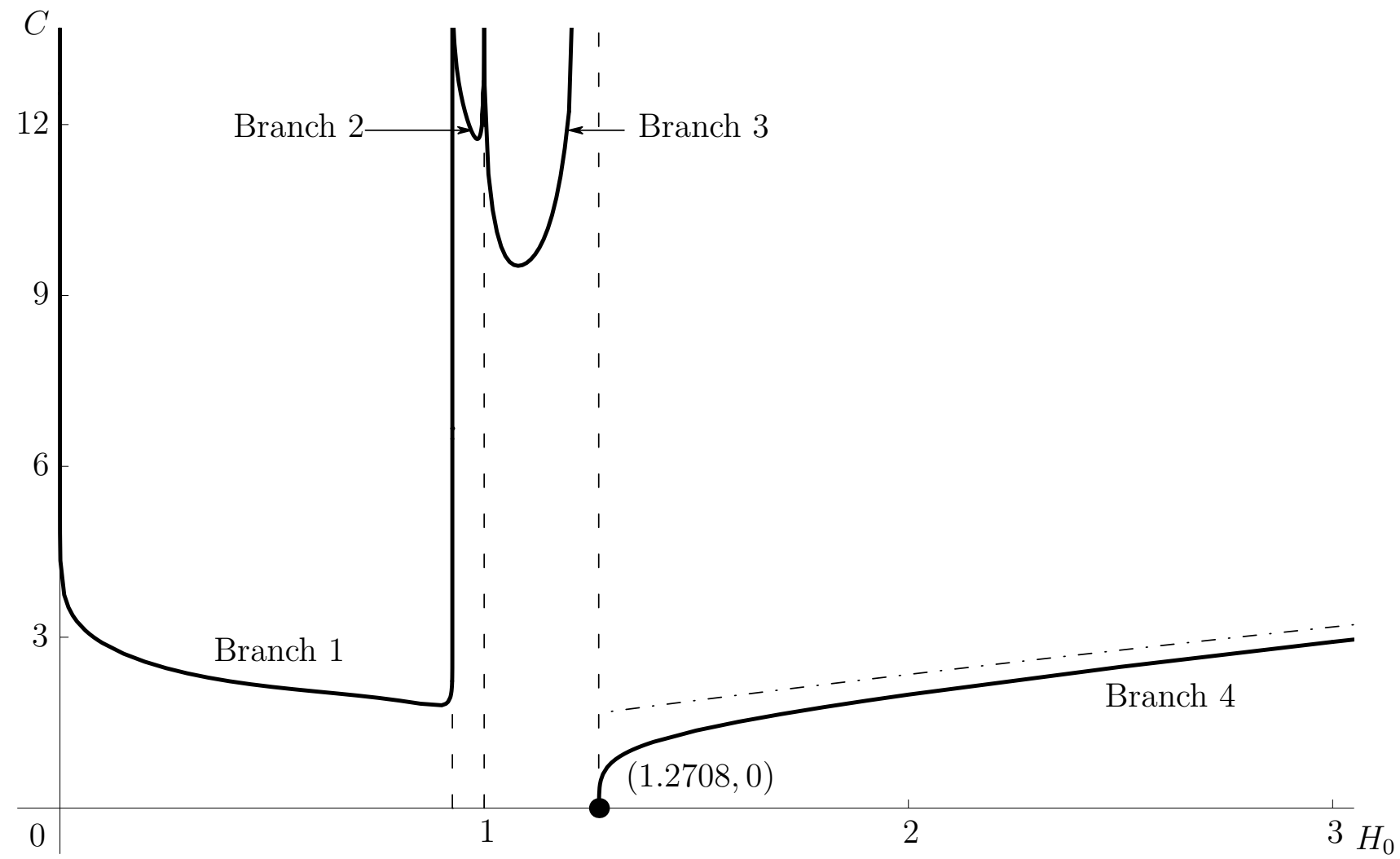

Figure 6: Plot of $C$ calculated from equation (41) as a function of $H_{0}$. The dashed-dotted curve is the leading-order asymptotic solution in the limit $H_{0} \rightarrow \infty$. 


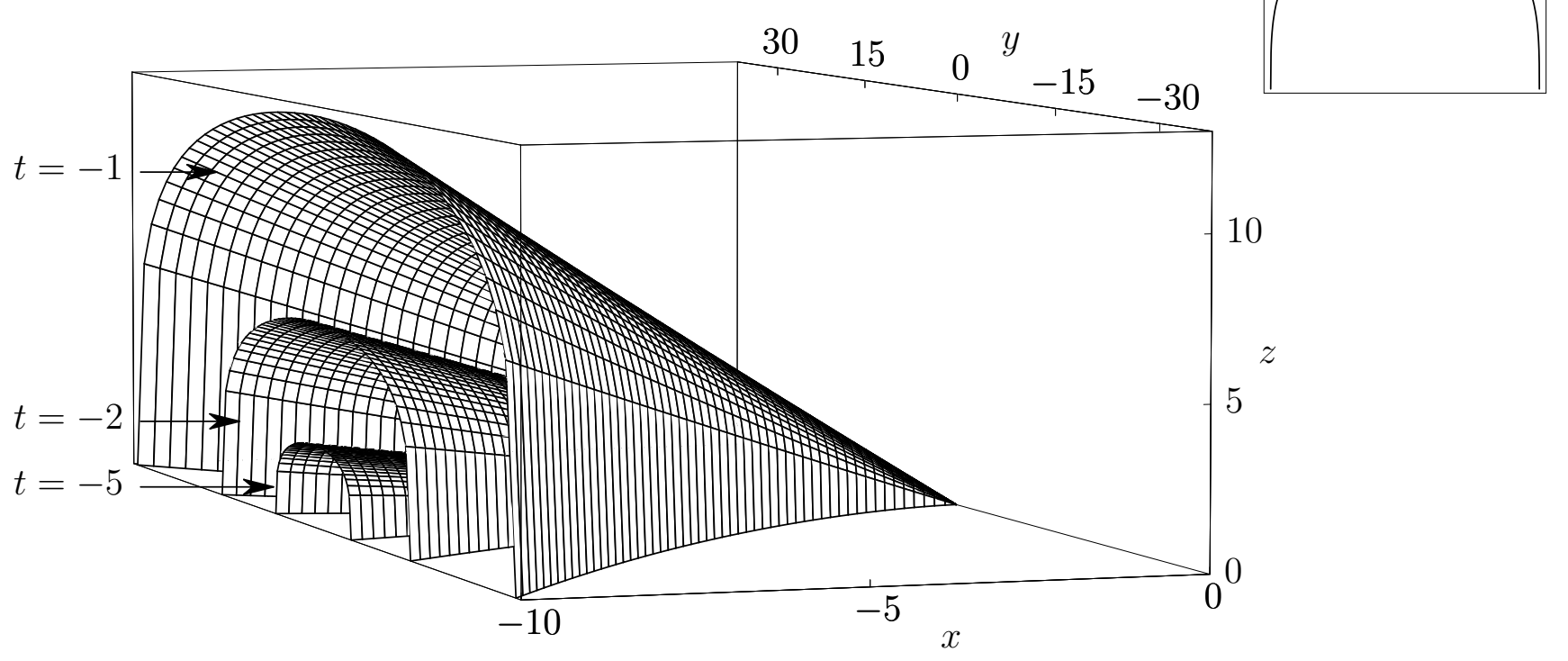

Figure 7: Three-dimensional plot of the free surface $z=h$ of the sessile rivulet predicted by the present similarity solution (23) with $H$ satisfying (36) subject to (25) and (26), for which $H_{0} \simeq 1.2708$ and $\eta_{0} \simeq 1.9152$, at times $t=-5,-2$ and -1 . The insert shows the cross-sectional profile. 


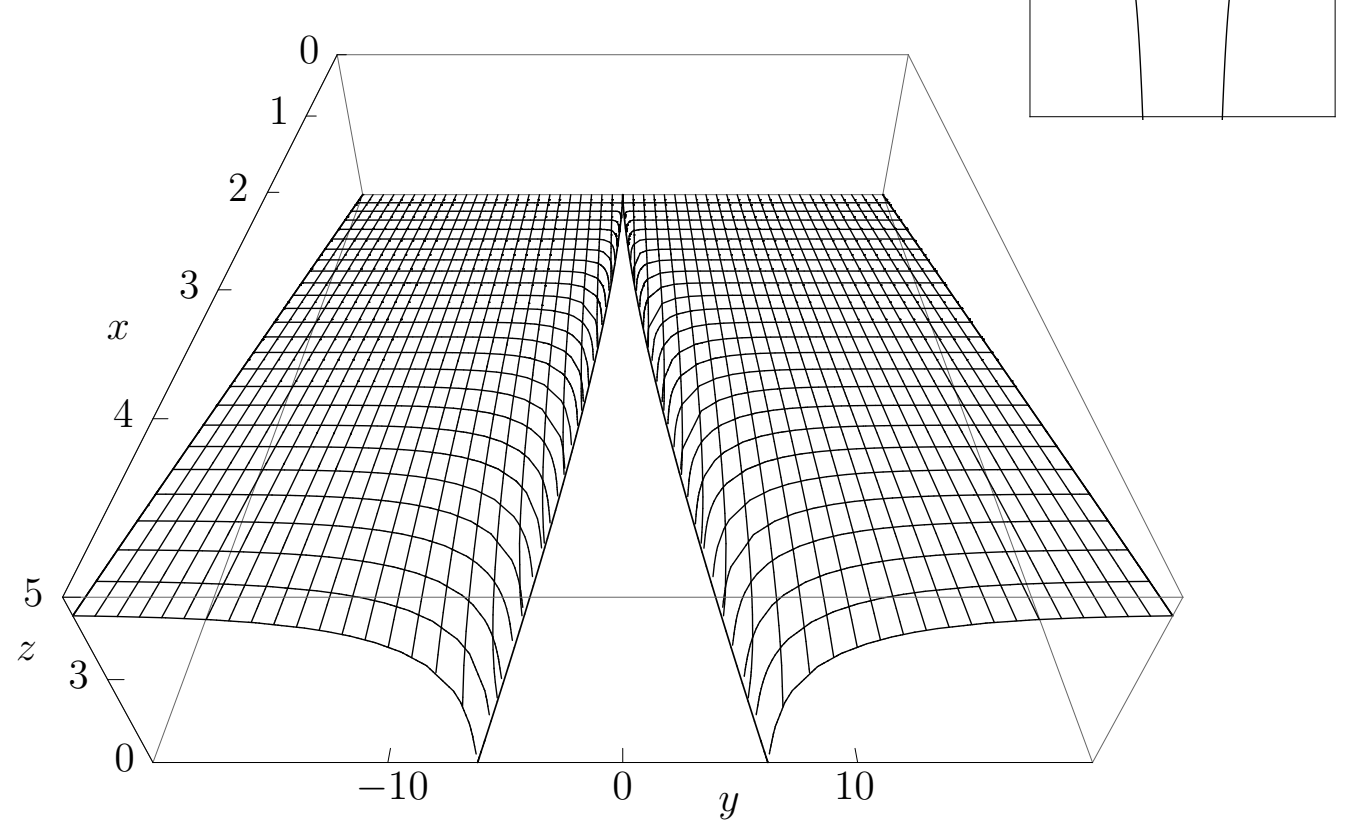

Figure 8: Three-dimensional plot of the free surface $z=h$ of the sessile film containing a dry patch predicted by the present similarity solution (23) with $H$ satisfying (49) subject to (26) and (44), for which $\eta_{0} \simeq 0.9573$, at time $t=1$. The insert shows the cross-sectional profile. 


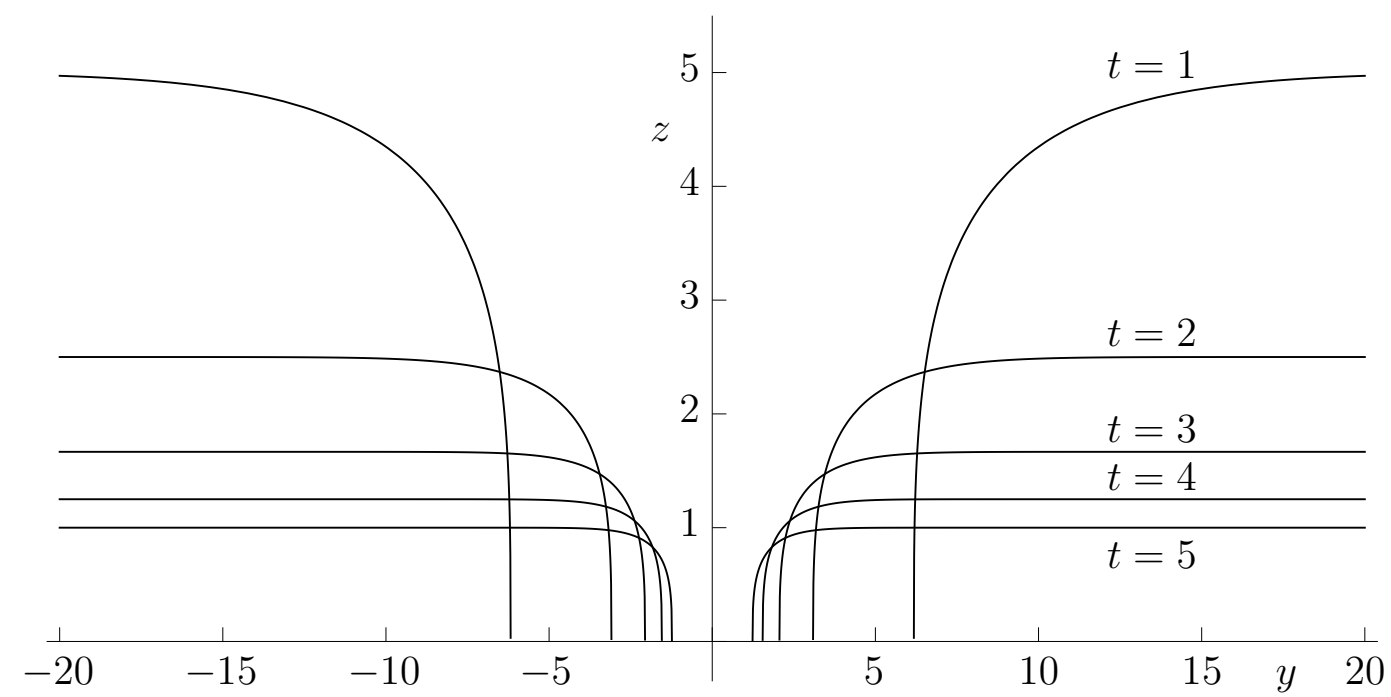

Figure 9: Cross-sectional profiles at position $x=5$ of the sessile film containing a dry patch, at times $t=1,2,3,4,5$. 